le portiQue Le Portique

Revue de philosophie et de sciences humaines

16 | 2005

Sartre. Conscience et liberté

\title{
La nature, entre métaphysique et existence :
}

Sartre et la question de la nature

\section{Laurent Husson}

\section{OpenEdition}

Journals

Édition électronique

URL : http://journals.openedition.org/leportique/733

DOI : $10.4000 /$ leportique.733

ISSN : $1777-5280$

Éditeur

Association "Les Amis du Portique"

\section{Édition imprimée}

Date de publication : 1 septembre 2005

ISSN : 1283-8594

\section{Référence électronique}

Laurent Husson, «La nature, entre métaphysique et existence : », Le Portique [En ligne], 16 | 2005, mis en ligne le 15 juin 2008, consulté le 25 mars 2021. URL : http://journals.openedition.org/leportique/ 733 ; DOI : https://doi.org/10.4000/leportique.733

Ce document a été généré automatiquement le 25 mars 2021.

Tous droits réservés 


\title{
La nature, entre métaphysique et existence :
}

\author{
Sartre et la question de la nature
}

Laurent Husson

1 Selon Merleau-Ponty, « une ontologie qui passe sous silence la Nature s'enferme dans l'incorporel et donne, pour cette raison même, une image fantastique de l'homme, sur le problème de l'esprit et de l'Histoire. Si l'on s'appesantit sur le problème de la nature, c'est avec la double conviction qu'elle n'est pas à elle seule une solution du problème ontologique, et qu'elle n'est pas un élément subalterne ou secondaire à cette solution ${ }^{1}$. Or, la philosophie de Sartre, non seulement est un anti-naturalisme (au double sens d'un refus de toute essence humaine ${ }^{2}$ et d'une déduction de l'homme à partir du non-humain), mais la question de la nature s'y trouve réduite, voire congédiée : elle n'est ni un principe explicatif, ni un objet autonome, ni un moyen de passage à un niveau ontologique différent. Dans la Critique de la raison dialectique ${ }^{3}$, la nature doit être traitée par la raison analytique sous peine d'une irrationalité profonde qui met en péril l'intelligibilité dialectique. En effet, au sein du mouvement dialectique, la nature est ce support qui totalise en extériorité le champ pratico-inerte. Elle introduit dans le monde le domaine de la «passivité » et de l'« inertie » ${ }^{4}$ et marque du sceau de l'extériorité la praxis humaine ${ }^{5}$.

2 Cependant, suivre cette ligne de réflexion conduirait à faire bon marché de l'une des deux perspectives explicitement tracées par L'Être et le Néant ${ }^{6}$, celle d'une "métaphysique de la nature ", en lien plus étroit au reste de l'œuvre qu'on ne l'imagine généralement, et à admettre sans examen que la Critique de la raison dialectique se déploie au même niveau d'enquête que le premier ouvrage. Mais quel est le sens de la formule «métaphysique de la nature»? Quels sont les enjeux de ce programme? Ne peut-on pas y lire l'opposition entre les démarches de Sartre et de Merleau-Ponty? Nous envisagerons tout d'abord la portée du refus de la dialectique de la nature dans la Critique de la raison dialectique, puis la manière dont l'ontologie du premier Sartre n'exclut pas les phénomènes naturels pour envisager à terme la manière dont peut se 
décliner le programme d'une métaphysique et ses conséquences quant à l'appréhension de la nature.

I. Le refus d'un rôle philosophique fondateur de la nature

3 La philosophie de Sartre se veut, dans un premier temps, une philosophie de la conscience s'inscrivant dans une tradition cartésienne et dans un horizon phénoménologique, qu'il aborde dans un souci critique concernant sa possible portée. Ce geste est repris dans la Critique de la Raison dialectique. L'horizon n'est plus la phénoménologie, mais le marxisme. Un des principes d'évolution de ce développement philosophique - depuis les premiers essais (La Transcendance de l'ego qui détache radicalement la conscience de tout ancrage naturel), jusqu'à L'Idiot de la famille consiste dans l'explicitation des médiations nécessaires pour comprendre le rapport concret de la conscience au monde et à elle-même. Ce trajet conduit d'une spontanéité absolue à une conscience incarnée et constituée dans la passivité, dont l'existence est la reprise de cette dernière ${ }^{7}$.

4 Ces deux horizons (la phénoménologie, le marxisme) traitent différemment la question de la Nature. Dans le cadre phénoménologique, la nature prend trois aspects : elle est le corrélat d'une couche première de l'expérience (comme dans la Crise des sciences européennes et la phénoménologie transcendantale ${ }^{8}$ ); elle renvoie à la multiplicité des modes d'être des existants non conscients (comme dans le deuxième tome des Ideen ${ }^{9}$ de Husserl ou dans Les Problèmes fondamentaux de la métaphysique ${ }^{10}$ de Heidegger); enfin, elle peut être envisagée dans le cadre de la détermination ontologique radicale de la totalité de l'étant, voire de son être. L'enjeu est alors le dépassement des formes classiques de l'ontologie. C'est probablement chez le dernier Merleau-Ponty que cette problématique s'est déployée de la manière la plus continue et avec le plus d'ampleur, dans le même champ que la pensée de Sartre. Dans le marxisme, au moment où Sartre écrit la Critique de la raison dialectique, l'idée d'une dialectique de la Nature est présente aussi bien chez les orthodoxes comme Roger Garaudy ${ }^{11}$ que chez des hétérodoxes, comme Henri Lefebvre ${ }^{12}$, même si c'est avec des statuts différents. De fait, la lutte contre cette menace dogmatique du matérialisme à marqué dès 1947, avec "matérialisme et révolution» le rapport de Sartre au marxisme ${ }^{13}$. Dans cette évolution, la réflexion sur la nature - hors du cadre étroit de la nature comme objet des démarches scientifiques analytiques ${ }^{14}$ et réduite à sa figure cartésienne (ou hégélienne ${ }^{15}$ ) d'extériorité pure et d'inertie - semble s'éloigner de plus en plus.

5 En effet si on envisage la première philosophie de Sartre, le thème de la nature est écarté du domaine de l'existence humaine, puisqu'il n'y a pas d'essence ou de nature humaine. Il n'apparaît que de manière incidente, au terme de l'Être et le Néant, avec l'évocation d'une hypothétique "métaphysique de la nature " ${ }^{16}$, laquelle n'est que le dernier mot d'un passage programmatique assez long, qui subordonne la réalisation de celle-ci à un certain nombre de questions. De plus, il semble que Sartre n'a jamais songé à poursuivre sa réflexion sur ce point. Dans la Critique de la raison dialectique, la question est celle d'une approche de la nature par le biais de la dialectique et d'une fondation de la réalité du mouvement dialectique dans la nature même, lui permettant ainsi d'envelopper l'histoire humaine. On aurait pu penser, en raison de la profession de foi matérialiste (par opposition à l'idéalisme hégélien) et dialectique, que le projet de L'Être et le Néant aurait pu être reformulé ou, du moins, que l'image donnée de la nature aurait pu se modifier. Or, l'introduction de la Critique de la raison dialectique comporte une critique méthodologique de l'idée de dialectique de la nature ${ }^{17}$. La critique d'Engels 
écartée, la position de Sartre se résume par l'alternative suivante. Si la dialectique se fonde dans la nature (comme objet des démarches scientifiques), elle devient une dialectique du dehors qui réduit l'histoire humaine à une forme de mécanisme et débouche sur un « discours sur l'idée de matière " ${ }^{18}$. Si, au contraire, l'histoire humaine n'est pas un mécanisme, il faut alors trouver une source à la dialectique, qui ne soit pas de l'ordre de la nature tout en n'étant pas un principe idéaliste. Il semble qu'on se soit fermé toutes les portes d'un matérialisme dialectique.

Or, pour Sartre, on résout la question, en dégageant, au sein même de l'être matériel, un être dont le mode d'être ne peut être compris qu'en fonction d'un acte de totalisation. Or, un tel mode d'être existe et n'est intelligible que pour une démarche dialectique. Cet être n'est rien d'autre que nous-mêmes en tant qu'être matériel : la praxis humaine. On aboutit à un « matérialisme réaliste, pensée d'un homme situé dans le monde, traversé par toutes les forces cosmiques et qui parle de l'univers comme ce qui se révèle peu à peu à travers une praxis en situation ${ }^{19}$. La Critique doit établir, par l'« expérience critique " ${ }^{20}$, l'extension de cette modalité de compréhension à partir du noyau fondamental qu'est la praxis individuelle dans le besoin à l'ensemble des praxis et des relations entre les hommes ${ }^{21}$. La dialectique ne trouve sa réalité et son champ d'expérience que dans le mouvement dialectique de la praxis humaine et non à partir de la nature considérée, soit dans certains de ses phénomènes (le passage de l'inerte au vivant) ${ }^{22}$, soit comme totalité (alors qu'elle est insaisissable comme telle, l'unité comme nécessité lui venant de la praxis humaine). Ce serait figer la dimension dialectique et le marxisme lui-même, comme le montre la réification non-dialectique de la dialectique telle qu'elle s'opère dans le stalinisme par la reprise dogmatique des efforts d'Engels ${ }^{23}$.

7 Ainsi, si on se place sur le plan des modes de fonctionnement de la raison, la nature et les phénomènes naturels relèvent d'un traitement "analytique », et non dialectique, sauf sur un plan heuristique, à titre de suggestion ${ }^{24}$. On ne peut appliquer aux phénomènes naturels la catégorie de totalité, pourtant essentielle pour la dialectique. La nature, par rapport aux phénomènes dialectiques, n'est qu'une gigantesque "décompression dispersive " ${ }^{25}$. De plus, les hypothèses dialectiques - possibles pour certains êtres naturels - restent des hypothèses car leur mode de vérification, qui s'inscrit dans la logique des sciences de la nature, demeure analytique ${ }^{26}$. On aurait donc un recul des prétentions par rapport à la fin de L'Être et le Néant, qui affirme la «métaphysique de la nature » comme une possibilité positive, alors même que le point de vue de la dialectique matérialiste pouvait renforcer un tel programme.

8 Cependant, le programme esquissé par la conclusion de L'Être et le Néant fournit des points de contact avec les exigences posées par la Critique de la raison dialectique: la nécessité, pour aboutir à une métaphysique de la nature, d'en passer par la question de l'action, et la référence à la notion de "forme", au sens où l'école de la "psychologie de la forme » a pu développer ce concept jusque dans le champ de la physique ${ }^{27}$. Or, Sartre utilise le même concept dans ses controverses avec les tenants de la dialectique de la nature pour indiquer qu'il se situe, non sur un plan dogmatique, mais sur un plan critique et heuristique ${ }^{28}$. Le modèle proposé par la psychologie de la forme est certes moins affirmé dans la Critique de la Raison dialectique ${ }^{29}$. Cependant, le contexte est différent : il s'agit de fonder la dialectique pour saisir des phénomènes sociaux, et non plus une ontologie ${ }^{30}$. Le champ ouvert par la Critique est plus étroit que celui de l'Être et le Néant, en amont ou en aval ${ }^{31}$. Les limites tracées par le second ouvrage ne permettent pas d'estimer les potentialités des traits fondamentaux de l'ontologie sartrienne. Il 
importe donc d'en revenir au texte de 1943 pour dégager les éléments qui permettent d'envisager des phénomènes autres que ceux de l'en-soi et du pour-soi et expliciter le programme de la conclusion de l'Être et le Néant. On pourra alors envisager la manière dont il peut se développer de différentes manières en fonction d'options métaphysiques différentes.

II. Du phénomène du mouvement au programme d'une métaphysique de la nature

9 Avant d'aborder le problème de la manifestation de la nature et de son expérience, il faut poser quelques préalables. Le premier est la nécessité de différencier, dans le cadre de l'ontologie de L'Être et le Néant, des niveaux structurels dans les différentes déterminations rencontrées aussi bien en ce qui concerne l'ordre du pour-soi que celui de l'en-soi.

ce qui concerne le pour-soi, cet aspect peut être mis en évidence de manière rapide en rappelant qu'il y a une différence entre l'ordre du pour-soi et celui du pour-autrui, l'un n'impliquant pas l'autre ${ }^{32}$. En ce qui concerne l'en-soi, le problème est plus complexe, car Sartre indique peu les différenciations dans l'usage qu'il fait de ce terme. La distinction la plus fondamentale est entre ce qui relève des conditions d'apparition d'un phénomène transcendant à la conscience ("l'être est, l'être est en soi, l'être est ce qu'il est ${ }^{33}$ ), les structures globales de l'apparition d'un monde (l'espace et l'extériorité, l'ensemble des catégories fixant la manière dont nous apparaissent les choses du monde), et ce qui y est irréductible. L'extériorité - négation de tout rapport entre les cecis du monde - n'appartient pas à l'en-soi comme tel, mais tel qu'il apparaît dans un monde. L'en-soi sartrien n'est donc pas assimilable à l'étendue cartésienne. Cependant, cette première différence n'est pas celle de l'être pur et de la nature. À la différence d'un réalisme classique, l'en-soi sartrien, que ce soit dans ses déterminations originelles ou sous sa configuration mondaine, ne peut avoir le statut d'un être créé, d'un naturé par rapport à un naturant (pour prendre des formules employées par Merleau-Ponty à propos de la conception chrétienne de la nature ${ }^{34}$ ). La seule dimension jouant un rôle créateur est celle du pour-soi. Or, ce dernier exige, au nom de la structure intentionnelle, le caractère non-créé de l'en-soi ${ }^{35}$. Lorsqu'il arrive à Sartre de dire que l'homme est la Vérité de la liberté divine et de son pouvoir créateur ${ }^{36}$, ces formulations ont un sens surtout moral.

11 L'analyse de la transcendance ${ }^{37}$ est un lieu privilégié du passage de l'être à la nature, c'est-à-dire à ce qui, dans la détermination des êtres singuliers, ne peut venir du poursoi sans se réduire, ni aux pures déterminations de l'en-soi, ni à celles du monde. Ici, une catégorie a toujours joué un rôle essentiel : le mouvement ${ }^{38}$. Chez Sartre, la reprise du phénomène du mouvement se fait en référence à la manière dont Bergson envisage l'aporie zénonienne de la flèche ${ }^{39}$. La perspective est cependant non bergsonienne, puisqu'il s'agit, non d'affirmer une irréductibilité de l'être par rapport à la pensée, mais l'irréductibilité d'un fait par rapport à l'être ${ }^{40}$.

Le contexte est celui de la "déduction ${ }^{41}$ des catégories de la transcendance du monde. En fonction du surgissement du pour-soi, les étants nous apparaissent selon les catégories kantiennes de la qualité, de la quantité, selon les catégories de l'essence et des puissances (depuis l'ordre de l'essence jusqu'à celui des probabilités), selon l'ustensilité (au sens heideggérien du terme). Sartre emploie les moyens de l'idéalisme (partir du pour-soi) pour établir un réalisme (pas de différence entre apparaître au pour-soi et être dans un monde). Ces catégories ne manifestent cependant pas l'être autrement que par l'indifférence sauf dans le cas des « abolitions et apparitions ${ }^{42}$ » (ou 
le changement ${ }^{43}$ ) et du mouvement ${ }^{44}$ (essentiellement le mouvement local), non déductibles des structures du pour-soi ${ }^{45}$. Elles ne peuvent faire l'objet que d'une "description ${ }^{46}$ qui en saisit ce que Sartre nomme leur «sens $"{ }^{47}$. Si la nature est, comme le veut Merleau-Ponty, l'expérience d'une «productivité qui n'est pas nôtre " ${ }^{48}$, ces catégories présentent un phénomène «naturel» qui ne tient, ni à une détermination interne affectant la transcendance de l'être, ni à la constitution d'une forme d'apparition de l'être, mais plutôt à un phénomène, se situant entre l'être et le non-être. On se concentre ici sur la question du mouvement ${ }^{49}$, car il manifeste un plus fort degré de contingence que l'«abolition » et l'« apparition " ${ }^{50}$ et est repris dans les « aperçus métaphysiques » des conclusions de L'Être et le Néant ${ }^{51}$.

Le problème posé par le mouvement d'un «ceci» (ou d'un objet) est celui du mode d'être du ceci en mouvement et de sa relation à l'espace. Décrire le ceci en mouvement implique de rompre avec deux caractéristique de l'être en soi d'un objet quelconque : la première est de dire qu'il est, la seconde qu'il entretient un simple rapport d'indifférence à l'espace. En effet, si le mobile ne peut être dit en mouvement ou en repos, c'est qu'il «n'est » pas, et que son être est un « être-de-passage » ${ }^{52}$. Or, cet « être de passage » se définit comme un redoublement du rapport d'extériorité à l'espace constitutif de la trajectoire du mobile. Ce dernier, en tant que "ceci», est dans un rapport d'extériorité à sa place, mais, comme on ne peut dire, ni qu'il " est », ni qu'il "n'est pas » à sa place au sens où il serait "ailleurs ", il faut admettre qu'il y "passe " lorsqu'il est en mouvement, il est extérieur à cette extériorité. Ce redoublement est un processus spécifique par quoi s'engendre une forme de synthèse, qui, pourtant s'évanouit aussitôt qu'elle s'engendre: la trajectoire. Le mouvement est donc placé entre l'être et le néant: le mobile est et n'est pas quelque part. Cette ambiguïté peut recevoir un sens car cette relation à l'espace s'approche de celle entre pour-soi et l'espace, lorsque ce pour-soi est considéré par autrui ${ }^{53}$. Cependant, cette relation ne se maintient pas par une néantisation continuée, mais qui en reste à la forme de la contingence d'un être.

14 Ainsi se manifeste à partir de l'en-soi - mais pour un pour-soi - quelque chose qui n'est, ni la simple coïncidence à soi de l'être, ni la dispersion totale de la pure extériorité, ni le surgissement d'un autre pour-soi (puisqu'il n'y a pas d'organisation d'une situation, de rapports spatiaux orientés en fonction d'une place et à partir d'un projet), ni le maintien de la trajectoire (puisque celle-ci se disperse en pure extériorité ${ }^{54}$ ). Il n'y a qu'un phénomène qui se situe entre les deux. Il peut avoir un sens sans qu'on puisse le fixer de manière nette, puisqu'il n'est pas saisi de manière interne, comme le pour-soi, mais sous la condition du surgissement au monde d'un pour-soi ${ }^{55}$.

15 Ainsi, même si on maintenait que la conception sartrienne de la nature extérieure se réduit plus ou moins à une conception cartésienne par figure et mouvement, il y a au moins une saisie de la nature irréductible à l'être, comme étant un fait contingent spécifique. De plus, au travers de la manière dont cette contingence affirme son irréductibilité et dont on essaie d'en fixer le sens par un rapport analogique, l'espace phénoménal qui s'ouvre ainsi est celui de l'ensemble des formes objectives d'irréductibilité. C'est ce dernier point que nous abordons en reprenant la manière dont Sartre situe ce qu'il appelle une «métaphysique de la nature».

Pour Sartre, le questionnement métaphysique renvoie à une "mise en question de l'existence de l'existant ${ }^{56}$, et plus exactement de la totalité de l'être, par un être qui est en question dans son être (le pour-soi, comme «mise en question de l'être par 
l'être »), et à partir d'un être ${ }^{57}$, ainsi qu'à la question de l'être comme totalité. La métaphysique est le domaine sur lequel ouvre l'ontologie. En effet, celle-ci est définie comme "l'explicitation des structures d'être de l'existant pris comme totalité " ${ }^{58}$ décrivant alors que la métaphysique est « l'étude des processus concrets et individuels qui ont donné naissance à $c e$ monde-ci comme totalité concrète et singulière ${ }^{59}$. La métaphysique de la nature est le point dernier de ce questionnement, en fonction de l'ordre suivant, chacune des étapes mettant en jeu le dépassement du dualisme ontologique de l'en-soi et du pour-soi, tel qu'il est posé dans l'introduction et tel qu'il se redéfinit au sein de l'ouvrage ${ }^{60}$.

1.- Le pour-soi est de lui-même relation à l'en-soi, mais il n'est pas l'en-soi tout en trouvant en lui son origine (comme l'indique la structure de la facticité et notamment la composante de la naissance ${ }^{61}$ ). La première question est donc celle de l'origine du pour-soi en tant qu'il est issu de l'être. Or, «l'ontologie se heurte à une contradiction profonde, puisque c'est par le pour-soi que la possibilité d'un fondement vient au monde $"{ }^{62}$. La métaphysique doit formuler des hypothèses sur le surgissement du poursoi : celles-ci doivent intégrer le problème du mouvement ${ }^{63}$, en unifiant ces deux surgissements.

2.- Le pour-soi fait surgir la seule forme concevable d'une totalité manquée en-soipour-soi, une "notion en perpétuelle désintégration ${ }^{64}$ qui pose la question de son statut ontologique et le problème de l'unité de l'être. Si on l'affirme, cet être a à la fois une dimension pour-soi et une dimension en-soi. Il est le "phénomène » et, « de ce point de vue, il n'y aurait qu'un phénomène: le monde » ${ }^{65}$, qu'il ne faut ni absorber dans l'immanence, ni dans la transcendance. C'est le point fondamental de la divergence entre Sartre et Merleau-Ponty, en même temps que la racine du traitement de la question de la nature.

3.- Le point essentiel du programme annoncé par Sartre est l'abord du problème de l'action. Selon la définition donnée dans la quatrième partie de L'Être et le Néant, agir est « modifier la figure du monde » ${ }^{66}$. Ici, il s'agit de modifier « l'être même » ${ }^{67}$ des choses singulières prises dans leur qualité. Le lien du pour-soi à l'être est alors modification de l'être à partir d'un être qui est au milieu du monde. Le statut ontologique du monde doit être déterminé pour saisir la signification de l'action, car le monde se modifie à partir d'un dépassement concret de l'intérieur du monde et par le biais d'un être au milieu du monde et des choses.

4.- La nature apparaît ici comme l'être qui se dévoile «par suite des répercussions de l'acte dans le monde ${ }^{68}$. La manifestation de l'être n'est plus un simple dévoilement. Les changements intra-mondains n'ont plus le sens de simples événements. Ils prennent, en analogie avec l'action, la forme d'un changement orienté de l'être par l'être. Dès lors, le maintien et le changement de certaines structures acquièrent une dimension de spontanéité en même temps que de cohérence. C'est ce qu'exprime la notion de forme gestaltiste à laquelle Sartre se réfère dans la description de la nature au terme de l'Être et le Néant, au début de la Critique, et d'un autre point de vue, dans les Cahiers pour une morale. En effet, le principe d'une Gestalt (ou structure) est que le tout est une structure à partir de laquelle les parties prennent leur valeur et leur sens. Les apports les plus incontestables de la Gestaltpsychologie ${ }^{69}$ se situent dans la psychologie de la perception, mais ces théoriciens ont également élaborée une théorie des formes physiques, pour laquelle les structures physiques manifestent des tendances à l'organisation dépassant le cadre du mécanisme. Dans les Cahiers, Sartre caractérise, par 
rapport au sens aristotélicien de forme, «la forme moderne (Gestalt)» [comme] unification du tout dans sa nature particulière et donc jusque dans sa substance ${ }^{70}$.

Sartre n'a pas développé ces indications. Ce programme est cependant très proche de celui d'un Merleau-Ponty. Pour celui-ci, la nature est en-deçà de la division sujet-objet et apparaît comme un "chiffre " (au sens donné par Karl Jaspers à ce terme) d'une indivision première, source pour des structurations ultérieures. Sartre, en renvoyant la nature au-delà de l'action, la subordonne à l'action et à l'histoire, ce qui conduit à une réaffirmation du sujet et à une philosophie de la personne ${ }^{71}$. C'est donc de la nature qu'il faudrait repartir ${ }^{72}$, pour retrouver l'être premier d'indivision d'où surgissent les significations. Mais quel serait, pour Sartre le statut de cette relation avec la nature?

On peut ici se guider sur une opposition entre ce que Merleau-Ponty nomme de manière programmatique "psychanalyse ontologique ${ }^{73}$ et un aspect particulier de la psychanalyse existentielle sartrienne, le dévoilement de la dimension significative des qualités et des choses, telle que la fin de l'être et le néant en analyse le principe, que Sartre retrouve chez le poète Francis Ponge ${ }^{74}$. Ce dernier, au travers des poèmes réunis dans le recueil Le Parti pris des choses ${ }^{75}$ donnerait les principes d'une " phénoménologie de la nature $"{ }^{76}$, une description de nos relations aux choses et de la manière dont nous y trouvons un sens, une fois opéré une " réduction phénoménologique » ${ }^{77}$ par rapport à toutes les idées préconçues de la science. Nous découvrons ainsi les êtres naturels et leurs qualités à partir du sens que le surgissement du pour-soi leur confère au sein de son projet d'être. Les choses acquièrent des significations de telle sorte que l'on est conduit à saisir la nature à partir de son coefficient d'étrangeté face au dévoilement humain. Les objets matériels ont des "manières de se comporter ${ }^{78}$. La perspective poétique de Ponge aboutirait à une forme de "caractéristique universelle " ${ }^{79} \mathrm{du}$ sens des choses pour les objets matériels. Cette dimension serait au fondement de tout rapport existentiel premier avec les êtres naturels, et, au travers d'eux, avec la nature elle-même.

La nature renvoie à la fois à la dimension d'irréductibilité de l'en-soi par rapport à son entrée dans le monde humain et au fait que l'homme, en transcendant l'en-soi, tend à se l'assimiler ${ }^{80}$ pour se donner, au travers de la chose, une image de l'en-soi-pour-soi. Elle n'entre donc dans le monde qu'à partir de ce projet comme projet d'appropriation, dont le projet de révélation (ou de connaissance) est un des modes. Un sens vient alors aux choses.

Ce sens, pour Sartre, est humain, mais pas uniquement subjectif pour autant. Une ambiguïté essentielle affecte la manière dont se manifestent les phénomènes naturels, ainsi que le montre, dans les Cahiers pour une morale, l'exemple de l'air liquide ${ }^{81}$. Les caractères de la nature sont marqués par l'ambiguïté d'une structure, configuration à la fois autonome et menacée d'extériorité simple, comme le montre le mouvement. On peut ainsi comprendre la Nature comme "développement figé d'une idée ». Cela signifie que les effets du mouvement tendent à demeurer dans une permanence, ou à produire une forme oscillant entre l'être et le néant. Celle-ci ne peut être ni absolument niée, ni absolument affirmée. L'idée de "décompression dispersive " ${ }^{82}$, qui renvoie de manière plus directe aux hypothèses métaphysique concernant le pour-soi lui-même comme " décompression d'être » ${ }^{83}$, marque cette oscillation.

Sartre ne nie donc pas que l'être ait des significations. Il ne renvoie pas l'expérience de l'émergence du sens à partir de l'être matériel et de ses qualités au subjectivisme pur et simple ou à une logique de la projection ${ }^{84}$. Cette émergence du sens révèle au contraire 
une dimension d'être et est porteuse de vérité. Il demande seulement de l'appréhender à partir de ses conditions de possibilité. De ce fait, il refuse de passer d'un monde humain à une nature sans les hommes, même s'il est vrai qu'il y a une reprise de l'homme par la nature. Poser cette condition n'est pas retourner au criticisme kantien ${ }^{85}$ car l'en-soi garantit la dimension de transcendance et nous évite de remonter au-delà de ce dernier et rien n'est ajouté à l'être par le surgissement du pour-soi ${ }^{86}$. On retrouve l'alternative métaphysique proposée, dans les conclusions de l'Être et le Néant entre le maintien de deux types d'être ou l'affirmation d'un troisième type d'être, le phénomène ${ }^{87}$. Celui-ci qui, transcendant les deux autres, serait à la source de la saisie de la nature dans sa consistance propre et comme productivité spécifique, puisqu'il serait possible d'envisager un lien fondamental entre la dimension en-soi et la dimension pour-soi sans passer par les conséquences de l'affirmation des structures du pour-soi comme " type d'être ", mais d'en intégrer certaines déterminations.

Ce programme peut sembler vague et verbal. Pour montrer qu'il est l'ouverture d'une véritable problématique dont dépend le statut de la nature, nous envisagerons, pour achever cette étude, comment se dessine, une fois exclues les formes inacceptables que sont le mécanisme classique (comme philosophie de la nature, et non comme pratique scientifique) et la dialectique de la nature, une alternative entre une métaphysique du phénomène et une métaphysique de la liberté.

III. Les deux voies de la métaphysique : du surgissement originel aux configurations naturelles

Dans ces "aperçus métaphysiques ", Sartre ouvre un champ de possibles ontologiques et détermine une condition ${ }^{88}$ qui permet justement d'exclure les deux métaphysiques (ils renvoient à une détermination totale de l'être et posant une affirmation, sur l'origine des êtres) que sont le matérialisme scientifique classique et la dialectique de la nature. Toutes deux oscillent entre l'immanentisme et le transcendantisme, ce qui conduit à la négation du problème qui doit être résolu : celui du surgissement de la liberté au sein de l'être. Ce sont donc les deux réponses possibles, maintien de la dualité conscience-être, affirmation du phénomène, dont nous allons envisager les formes et les significations aux quatre niveaux du questionnement métaphysique dégagés plus haut.

28 a) Pour le premier niveau, Sartre, dans l'analyse des pures structures du pour-soi a développé l'hypothèse d'un projet de l'en-soi pour se fonder, ce qui fait de l'être une "aventure individuelle ${ }^{89}$. Cette aventure aboutit au surgissement du pour-soi facticiel, c'est-à-dire ayant en lui une dimension non fondée. Cette dimension de facticité se décline ensuite sous plusieurs formes, dont le corps, par exemple, est une des plus fondamentales. Or, si on maintient une dualité de structure, la facticité est simplement le noyau de la finitude du pour-soi et sa forme simplement «la forme contingente que prend la nécessité de ma contingence ${ }^{90}$. Les déterminations de cette facticité s'enrichiront, ainsi que la relation à l'en-soi, au fur et à mesure qu'on envisagera le pour-soi selon des registres de plus en plus concrets.

$\mathrm{Au}$ contraire, la position du phénomène implique de réinscrire cette facticité dans une totalité première, enveloppant ainsi le pour-soi au milieu du phénomène par la réversibilité du rapport voir/être-vu (par les choses). La facticité est saisie à partir de la forme de son insertion dans le monde, et non à partir de la contingence. Elle devient potentiellement porteuse $d u$ sens du phénomène, par exemple dans le fait que le toucher puisse faire preuve de réflexivité, et, de manière plus générale, que le voyant 
devienne visible, ce que Sartre renvoie à l'ordre de la métaphysique ou de l'anthropologie ${ }^{91}$. Si nous réinterprétons la problématique de Merleau-Ponty selon la perspective de Sartre, elle s'inscrit dans ce cadre par le fait même de donner un sens et un rôle spécifique à certaines structures corporelles. Cette ambivalence profonde marque bien comment la démarche qui veut reconnaître dans le naturel (ce qui est lu par la science selon le registre du biologique) un chiffre ontologique est liée à un choix métaphysique originaire, qui se fait antérieurement à la détermination ontique de ce corps. La problématisation sartrienne n'est pas simplement une coupure avec l'être de telle sorte qu'on aboutisse à une autoposition absolue. Elle permet de problématiser le sens de cette relation qui se compliquera au fur et à mesure de la concrétisation de ces structures, en même temps que se développera l'investissement à partir du monde dès la naissance - de ma propre facticité.

b) Le second niveau est celui de la pure transcendance. Son analyse clôt celle des structures fondamentales du pour-soi et dégage la structure de monde (phénoménologique) de la manifestation de l'être. La question de la nature n'y est pas encore posée explicitement, mais on passe de la consistance en soi absolue de l'être à la dimension de l'extériorité spatio-temporelle et, au sein de cette dimension, on rencontre ce qui peut être compris comme venant de l'être, et non de nous: sa présence pure qu'est la qualité ${ }^{92}$, l'abolition-apparition ${ }^{93}$ et le mouvement. L'interprétation de ces déterminations dépend elle aussi du choix ontologique opéré.

31 En effet, l'interprétation métaphysique sartrienne par l'effort de fondation (le mouvement comme "première "tentative" de l'en-soi pour se fonder et "maladie de l'être" ${ }^{94}$ ) s'opère avec les moyens du bord. Sartre reste dans l'ontologie et ne dispose donc que du double cadre de l'en-soi comme origine et du pour-soi se vivant comme effort de fondement, ainsi que de l'articulation en terme d' « image " ${ }^{95}$ des structures du pour-soi et de sa configuration en monde. Cependant, si le choix est fait d'envisager le «monde » comme structure unique ${ }^{96}$ et de le comprendre comme manifestant un originaire, ainsi que le fait Merleau-Ponty, la question du mouvement ne se pose plus dans son excès par rapport à l'en-soi et au pour-soi, mais par rapport à l'ensemble des structures et des moments du monde lui-même, au sein d'un processus ayant à la fois une part d'immanence et de transcendance. Cette structure présente une esquisse d'auto-configuration en deçà de toute constitution, qui renverrait, pour Merleau-Ponty (quoique celui-ci ne traite pas directement le problème) vers un en-deçà de la scission en-soi/pour-soi et exprimant une dimension propre de l'être. Or, s'il est vrai que la nature est l'expérience de ce type de productivité émergente par rapport à l'extériorité, il y a bien un sens du phénomène du mouvement, l'expression d'une structure, alors que Sartre, tout en reconnaissant le phénomène dans son irréductibilité, le saisit d'abord dans l'expérience d'une contingence spécifique au sein de la structure de la manifestation.

Par rapport aux projets du dernier Merleau-Ponty, ces deux premiers niveaux renvoient au premier point indiqué dans le dernier plan des notes de travail de le Visible et l'Invisible, à savoir: " I. Le visible $"{ }^{97}$. Le second point, " II. La nature ${ }^{98}$, renverrait de manière plus explicite aux deux niveaux suivants.

c) Le troisième niveau est celui la situation (telle qu'elle est explicitée dans la quatrième partie de l'Être et le Néant) et de l'action (dans plusieurs passages des Cahiers pour une morale) comme entrée réciproque du pour-soi et des cecis singuliers au milieu du monde. Le pour-soi intervient comme en-soi au sein de l'être en raison même de son 
rapport fondamental à l'être ${ }^{99}$ et l'en-soi se dévoile dans la fragilité de son être, sa Gestalt ou sa structure matérielle. La nature se dévoile au travers de la manière dont le pour-soi la dévoile, voire "crée ce qui est ${ }^{100}$. Ce dévoilement, lié à l'action, nous confronte à des qualités et des structures naturelles. La question des hypothèses métaphysiques se pose dans l'analogie entre la relation d'être intentionnelle de l'en-soi à lui-même par le biais de l'action et la relation d'être non-intentionnelle des structures naturelles ${ }^{101}$ et de leurs métamorphoses. Dans l'action, la matière se révèle dans sa diversité et dans ce qui peut surgir comme sens à partir d'elle ${ }^{102}$, en présentant une ambiguïté essentielle: forme neuve, émergente et irréductible ou bien simple réarrangement d'atomes.

Pour Sartre, la présence d'une structure (Gestalt) est plus utilisée en liaison avec l'affirmation d'une création humaine mais, pour Merleau-Ponty, cette présence est le signe d'une dimension pré-objective de l'être, d'un excès par rapport à toute réduction productrice ${ }^{103}$ se manifestant dans une auto-organisation. Merleau-Ponty essaie d'y saisir le phénomène de Gestalt au-delà de l'opposition immanence-transcendance ${ }^{104}$. Cette opposition est fondée sur l'interprétation du phénomène de monde. En effet, si nous continuons ici à comprendre l'ontologie recherchée par Merleau-Ponty comme reposant sur l'affirmation de l'irréductibilité du phénomène de monde, la possibilité du traitement des phénomènes intramondains a pour sens de manifester justement l'irréductibilité, pour l'être, d'un traitement tout en transcendance ou tout en immanence. La Gestalt renvoie à l'idée de strate de l'être à déplier. Pour Sartre, cette dimension spécifique est certes une limite imposée à l'extériorité, mais en tant que la nature se dévoile au travers de l'action humaine, c'est-à-dire au sens où la forme, analogue au dévoilement-création humain, ne renvoie pas au-delà de l'inertie première de l'en-soi comme à une nouvelle dimension, mais affirme une contingence spécifique, une ambiguïté au sein de celle-ci. Alors que, pour Merleau-Ponty, l'ontologie doit recommencer à partir de ce point, elle s'y arrête pour Sartre. Elle laisse alors la place, soit à la métaphysique, soit à la démarche scientifique formulant ses propres exigences, soit encore l'analyse existentielle. Dans chacun de ces trois cas, l'ontologie a un rôle à jouer : situer le statut de la métaphysique et lui fournir les données par lesquelles elle peut s'élaborer, poser les conditions de la relation à l'être sur laquelle se fonde la démarche scientifique, la raison dialectique situant cette démarche au sein d'une dialectique de la raison avec elle-même et la métaphysique n'ayant qu'une vocation herméneutique, dégager l'origine vraie des significations rencontrées dans l'existence humaine. Néanmoins, parce qu'on se trouve ici au sein de l'être, parce que l'être-en-soi se manifeste à l'origine de sa propre configuration, le pour-soi n'ayant introduit dans le monde que l'activité-passivité et le sens de la totalité, il y a bien ici une nature.

d) Le dernier niveau est celui du rapport existentiel à la nature, notamment matérielle ${ }^{105}$. Ce rapport existentiel qui vient au monde par la dimension en-soi du pour-soi, hante tout rapport de l'en-soi avec lui-même. Deux aspects de cette relation peuvent être pris en compte. Le premier est la façon dont le "projet appropriatif " analysé dans L'Être et le Néant ${ }^{106}$ nous amène à faire l'expérience, au sein de l'être même des choses, de significations nouvelles, humaines au sein de l'être. La "psychanalyse des choses » ${ }^{107}$ et la relation à l'être au fondement de toute la signification globale des qualités à partir desquelles nous sommes amenés à découvrir des valeurs se situe ici. Le second aspect serait celui de la création artistique, que celle-ci soit poésie, comme Ponge, ou sculpture, comme chez Calder ${ }^{108}$. Celles-ci, au-delà d'un être, peuvent nous révéler la totalité de l'être et nous rendre sensible l'ambiguïté de la nature. Mais, là 
encore, le choix métaphysique joue son rôle. Avec l'affirmation du phénomène, le dévoilement met en jeu des significations par lesquelles nous ferons partie de l'être. Les fonder aura pour enjeu de contribuer à enrichir la dimension «verticale " ${ }^{109}$ de l'existence. Inversement, en rester à la dualité ontologique des types d'être nous renvoie à la découverte par l'homme de sa situation au sein de l'être, en tant que l'être lui renvoie une image de lui-même. Cette image est celle d'un monde où «les choses sont humaines " ${ }^{110}$, ont une "profondeur indifférenciée où sont mélangés mille possibles indistincts et humains » ${ }^{111}$, ce qui, pour Sartre, fait le sens profond de toute expérience du sens au travers de la nature. Mais cette découverte est ambiguë, débouchant moins sur l'origine que sur une possible aliénation ${ }^{112}$ si l'homme ne déchiffre qu'un destin et non la situation de son aventure.

Conclusions

La problématique de la nature n'est ni occultée, ni rendue impossible par la philosophie de Sartre. Le rejet de la dialectique de la nature ne conduit pas à une affirmation dogmatique du mécanisme et le caractère succinct des hypothèses de L'Être et le Néant ne les rendent pas invalides. Dans l'ontologie, il n'y a pas simplement l'être et la conscience. Le monde y est bien présent et même les animaux n'y sont pas impossibles. Il y a un effort de Sartre pour dégager des critères d'acceptabilité et pour situer le choix de sa thématisation philosophique à son ordre et à sa place. Sartre n'a pas suivi la voie d'un Merleau-Ponty, mais la notion qu'il développe, et de la métaphysique, et des phénomènes naturels permet d'en comprendre les raisons. L'affirmation de l'indivision originelle de l'être lui semble un élément qui relève d'une option métaphysique, par rapport à laquelle Sartre a été réticent par rigueur philosophique. Sartre - cartésien en cela - n'a pas voulu supposer plus de principes que ceux dont il avait besoin. Enfin, l'affirmation de la contingence n'a jamais été pour lui le signe de l'échec de la pensée, mais la marque de sa reconnaissance du réel et de son lien propre avec ce dernier. En ce sens, s'il n'y a pas de philosophie de la nature au sein de l'ontologie ou comme moment fondamental de celle-ci afin de la dépasser, mais une "métaphysique de la nature », ce n'est pas au sens où celle-ci s'avère le chiffre d'un originel absolu, mais celui d'une forme particulière de la contingence, susceptible d'hypothèses au sein du domaine de la liberté.

\section{NOTES}

1.. M. MerleAu-Ponty, La Nature. Cours du collège de France, Paris, Seuil, « Traces écrites », 1995, p. 355.

2..Voir J.-P. SARTRE, L'Existentialisme est un humanisme, Paris, Nagel, 1946, repris dans la collection « Folio-essais », présentation et des notes d'Arlette Elkaïm-Sartre, 1996 (noté $E H$ dans la suite, les références allant à cette dernière édition). Sartre développe ce qu'il appelle le premier principe de l'existentialisme : « L'homme est d'abord un projet qui se vit subjectivement, au lieu d'être une mousse, une pourriture ou un chou-fleur » (EH, p. 30). Dans ce cadre, la question de la nature n'est même pas posée, puisque le pendant de la comparaison est moins la nature que le schéma créationnisme (les choses 
apparaissent comme créées en fonction d'une essence, et réalisent cette essence). Sartre polémique ici contre les catholiques pour qui la création est ce par quoi les choses sont assignées en quelque sorte par Dieu à leur essence.

3.. Jean-Paul SARTRE, Critique de la raison dialectique, tome 1. Théorie des ensembles pratiques, Paris, Gallimard, « Bibliothèque des Idées », 1960, texte établi par Arlette Elkaïm-Sartre, Paris, Gallimard, «Bibliothèque de philosophie », 1986 (noté CRD, I dans la suite), tome 2 (inachevé). L'intelligibilité de l'histoire, texte et glossaire établi par Arlette Elkaïm-Sartre, Paris, Gallimard, 1985 (noté CRD, II dans la suite). Nos références vont à ces dernières éditions.

4.. Voir la description de la dialectique première du besoin, CRD, I, p. 200-201. « La pluralité inerte devient totalité pour avoir été unifiée par la fin comme champ instrumental, elle est en elle-même la fin tombée dans le domaine de la passivité. Mais loin que son inertie nuise à son caractère de totalité faite, c'est elle qui le supporte ». 5.. Voir CRD, I, p. 445 : « le champ pratico-inerte n'est pas un nouveau moment d'une dialectique universelle mais la pure et simple négation des dialectiques, par l'extériorité et la pluralité ». Nous n'identifions pas le pratico-inerte et la nature au sens où nous l'avons caractérisée, mais la dimension « antidialectique » du praticoinerte se fonde sur la dimension non-humaine du champ pratique unifié par la dialectique constituante, telle qu'elle prend sens à partir de la liberté humaine. Mais cette manière de prendre sens, lorsqu'il s'agit d'un champ naturel, manifeste aussi pour la nature un sens.

6.. Jean-Paul SARTRE, L'Être et le Néant, Paris, Gallimard, « Bibliothèque des Idées » , 1943, repris en 2001 dans la coll. « Tel », éd. Arlette Elkaïm-Sartre (noté $E N$ dans la suite). Nos références vont à cette dernière édition.

7.. On suit ici la thèse de Vincent de COOREBYTER développée à la fin de son introduction à son édition de La Transcendance de l'ego, Paris, Vrin, « Textes et commentaires », 2004, p. 75-76 notamment.

8.. E. HUSSERL, La Crise des sciences européennes et la phénoménologie transcendantale, traduction française par Gérard Granel, Paris, Gallimard, « Bibliothèque de philosophie », 1976.

9.. Idées fondamentales pour une phénoménologie et pour une philosophie phénoménologique, tome II, Recherches phénoménologiques pour la constitution, traduction française par Éliane Escoubas, Paris, PUF, « Épiméthée », 1982.

10.. Martin HEIDEGGER, Concepts fondamentaux de la métaphysique. Monde-finitude-solitude, traduit de l'allemand par Daniel Panis, Paris, Gallimard, «Bibliothèque de philosophie » , 1992.

11.. Au moment de la publication de la Critique de la raison dialectique et du débat qui s'en ensuivit, Roger Garaudy était membre du bureau politique du Parti Communiste Français, directeur du Centre d'Études et de Recherches marxistes. Il est un des contradicteurs de Sartre dans le volume collectif, Marxisme et existentialisme. Controverses sur la dialectique par Jean-Paul SARTRE, Roger GARAUDY, Jean HYPPOLITE, Jean-Pierre VIGIER, J. ORCEL, Paris, Plon, « Tribune libre », 1962. Ce volume est la retranscription d'un débat sur la dialectique de la nature.

12.. Voir Henri LEFEBVRE, « Critique de la critique non critique », Nouvelle revue marxiste $\mathrm{n}^{\circ} 1$, repris partiellement dans Métaphilosophie, Paris, Éditions de Minuit, " Arguments », 1964, Syllepse en 2000, préf. Georges Labica. La critique de H. Lefebvre par rapport à la dialectique de la Nature se situe aux pages 84-86 de cette dernière 
édition. Elle dogmatise les réflexions de Sartre sur la dialectique de la nature en disant que "Sartre en vient à rayer purement et simplement l'existence de la nature » (p. 84). 13.. Voir Jean-Paul SARTRE, Situations III, Paris, Gallimard, 1949, p. 135-225, notamment les pages 144-157.

14.. C'est ainsi qu'elle est notamment appréhendée au début de la Critique de la Raison dialectique.

15.. S'il arrive à Sartre d'évoquer l'extériorité cartésienne (voir $E N$, p. 628, où l'extériorité est mise en rapport avec la spatialité), mais la référence la plus probable est l'extériorité hégélienne (voir " Matérialisme et révolution », Situations III, p. 154, " Marxisme et existentialisme. controverse sur la dialectique », op. cit. : « Ainsi Hegel a pu dire que la Nature est extériorité » (p. 14)).

16.. EN, p. 673.

17.. CRD, I p. 145-155 notamment.

18.. CRD, I, p. 148.

19.. CRD, I, p. 148.

20.. Voir CRD, I, p. 164-165 et, plus généralement le point $B$ de l'introduction.

21.. Voir CRD, I, p. 156 : « ...la praxis de tout mouvement dialectique doit se découvrir à chacun comme la nécessité de sa propre praxis et, réciproquement, que la liberté en chacun de sa praxis singulière doit se redécouvrir en tous pour découvrir une dialectique qui se fait et le fait en tant qu'elle est faite ».

22.. Voir CRD, p. 152, note.

23.. Voir CRD, I, p. 145 : « Cette difficulté [de fonder la dialectique] a paru insurmontable aux marxistes d'aujourd'hui; ils n'ont vu qu'un seul moyen de la résoudre: refuser à la pensée elle-même toute activité dialectique, la dissoudre dans la dialectique universelle, supprimer l'homme en le désintégrant dans l'Univers. Ainsi peuvent-ils substituer l'Être à la Vérité. Il n'y a plus à proprement parler de connaissance, l'Être ne se manifeste plus, de quelque manière que ce soit: il évolue selon ses lois propres; la dialectique de la nature, c'est la nature sans les hommes ».

24.. Sartre demeure à cet égard dans la Critique de la Raison dialectique sceptique, en raison de l'absence de contradiction interne aux procédures analytiques de la science : «Pour l'instant, la biologie, dans le domaine concret de ses recherches demeure positiviste et analytique. Il se peut qu'une connaissance plus profonde de son objet lui donne, par ses contradictions, l'obligation de considérer l'organisme dans sa totalité, mais cela n'est pas sûr » (CRD, p. 152-153).

25.. CRD, I, p. 198.

26.. Voir CRD, I, p. 151: « Faut-il donc nier l'existence de liaisons dialectiques au sein de la Nature inanimée ? Nullement. À vrai dire, je ne vois pas que nous soyons, dans l'état actuel de nos connaissances, en mesure de nier où d'affirmer [...] ; de toute façon, dans le domaine des faits de la Nature inorganique, il s'agira d'une affirmation extrascientifique ».

27.. Voir pour ce point, l'exposé classique fourni dans le deuxième chapitre de Paul GUILlaume, La Psychologie de la forme, Paris, Flammarion, «Bibliothèque scientifique ", 1937, p. 27-28: « Mais il existe aussi des faits physiques dont les parties ne restent pas identiques à elles-mêmes dans leur groupement et qui possèdent les caractères des formes [...]. $1^{\circ}$ Ensemble statiques en équilibre où aucun changement ne s'est produit pendant un temps assez long [répartition d'une charge électrostatique dans un diélectrique $; 2^{\circ}$ ) Processus stationnaires; il s'agit de changements à allure régulière, 
continue ou périodique (ondes produits par un choc ou diapason [...], passage du courant électrique, etc. ; $3^{\circ}$ ) Processus quasi-stationnaire ».

28.. Voir Marxisme et existentialisme. Controverses sur la dialectique, op. cit., p. 13, à propos de l'usage de la notion de forme comme possible en biologie.

29.. Voir CRD, I , p. 153, la défense de Kurt GOLDSTEIN, dont Sartre a édité, dans la collection qu'il dirigeait chez Gallimard, La Structure de l'organisme (traduction française de E. Burchkart et J. Kuntz, Paris, Gallimard, « Bibliothèque de philosophie », 1951). Si cet auteur ne se situe pas directement dans le cadre de la psychologie de la forme, il rejoint celle-ci sur plusieurs points, notamment sur le primat du tout par rapport aux parties.

30.. Voir la remarque dans le deuxième tome de la Critique de la raison dialectique, à propos de l'étude de « l'être-réel » (CRD, II, p. 311) de la totalisation d'enveloppement comme étude de «l'unité dialectique de l'humain et de l'anti-humain sous toutes leurs formes et en tant qu'elle repose sur l'unification pratique de l'extériorité physicochimique (et zoologique) en milieu (plus tard en champ) par l'organisme et sur le reconditionnement de l'organisme par le physico-chimique à travers l'indispensable unité synthétique du milieu et du champ ; ce problème difficile ressortit à une ontologie de l'Histoire et non pas à une critique de la Raison dialectique » (CRD, II, p. 311-312). 31.. On comprend le premier ouvrage de Sartre comme n'ayant pour objet que le dégagement des structures ontologiques de la condition humaine. Or, outre le fait que l'Être et le néant n'est pas une anthropologie (ce que Sartre reconnaît lui-même dans les Cahiers pour une morale), il importe de se rappeler que le problème initialement posé au terme de l'introduction de l'ouvrage de 1943 est celui d'une conception totale et unifiée de l'Être (cf. EN, p. 33).

32.. Voir $E N$, p. 336 : « l'existence des autres n'est pas [...] une conséquence des structures ontologiques du pour-soi ». On pourrait retrouver d'autres différences plus fines au sein des structures du pour-soi, mais le traitement d'un tel problème implique une relecture de L'être et le néant que nous ne pouvons pas entreprendre ici.

33.. EN, p. 33.

34.. Voir M. MERLEAU-PONTY, La Nature, op. cit., p. 26 : « À partir de ce moment [de la tradition judéo-chrétienne], la nature se dédouble en un naturant et un naturé ». 35.. Voir EN, p. 31.

36.. Voir « La liberté cartésienne », Situations I, Paris, Gallimard, 1947, p. 308.

37.. EN, p. 207-255. Ce terme renvoie ici aussi bien à la transcendance husserlienne qu'au sens heideggérien du terme, notamment la manière dont il est développé dans De l'être-essentiel d'un fondement ou " raison ", traduction Henry Corbin, repris dans Questions I, Paris, Gallimard, « Les Classiques de la philosophie », 1968.

38.. $E N$, p. 245-250.

39.. Voir Henri BERGSON, par exemple « la perception du changement ", dans La Pensée et le Mouvant, repris dans Euvres, éditions du Centenaire, André Robinet (éd.), Paris, PUF, 1959.

40.. La problématique de Sartre est métaphysique et ne relève pas d'une phénoménologie de la perception (même si la question du mouvement est envisagée après l'espace, elle l'est avant la constitution du corps propre), comme on peut le voir chez Husserl dans Chose et espace. Leçons de 1907, introduction, trad. française et notes de Jean-François Lavigne, Paris, PUF, 1989, sixième section « La constitution du changement objectif », XVI - La constitution du simple mouvement, p. 324-328. Chez Husserl, c'est la coordination kinesthétique qui donne la signification du « en 
mouvement » ou du « en repos », compte non tenu d'éléments supplémentaires liés au déplacement du corps-propre par un objet et dont la correction doit être apportée par l'interprétation d'un ensemble d'éléments kinesthétiques en rapport.

41.. Voir $E N$, p. 246, l'opposition de la « déduction » et de la « description ».

42.. EN, p. 243.

43.. Voir $E N$, p. 243-245.

44.. EN, p. 243-250.

45.. On retrouve ici une position kantienne, quoique dans un contexte différent. En effet, pour Kant, « le mouvement d'un objet dans l'espace n'appartient pas à une science pure, ni par conséquent, à la géométrie ; car que quelque chose soit mobile, cela ne peut être connu a priori, mais seulement par expérience (Emmanuel KANT, Critique de la raison pure, Analytique transcendantale, tr. fr., in Kant, Euvres, tome I, Paris, Gallimard, «Bibliothèque de la Pléiade ", 1980, p. 869 note).

46.. EN, p. 246. Sartre évoque à propos de l'abolition et de l'apparition une opposition entre une " élucidation ontologique » et une « élucidation métaphysique » (EN, p. 243). Nous y reviendrons ultérieurement.

47.. EN, p. 246.

48.. M. MERLEAU-PONTY, La Nature, op. cit., p. 19.

49.. Cette question a été peu approfondie par les commentateurs. On citera l'étude qu'en fait Gerhard SEel dans La Dialectique de Sartre, Bonn, Bouvier, 1971, tr. fr., L'Âge d'homme, «Raisons dialectiques ", 1995, 2.3.4 La solution gnoséologique du problème de la temporalité mondaine, p. 193-197, et plus particulièrement les pages 205, 209-214. L'étude la plus approfondie est celle de Bernard BESNIER, « Le problème du mouvement ", dans J.-M. MouILLIE, (éd), Sartre et la phénoménologie, Fontenay-aux-Roses, ENS Éditions, « Théoria », 2000, p. 175-209.

50.. « Un monde sans mouvement serait concevable. Certes, on ne saurait envisager la possibilité d'un monde sans changement, sauf à titre de possibilité purement formelle, mais le changement n'est point le mouvement » (EN, p. 245).

51.. Voir EN, p. 669.

52.. EN, p. 247.

53.. Voir la référence au mouvement dans l'analyse de la perception du corps d'autrui, $E N$, p. 388, où l'évocation de l'irréductibilité du mouvement est un argument pour la réfutation de l'identification du corps vivant au cadavre, le corps vivant étant un cadavre qui bougerait.

54.. Voir EN, p. 250 : « la ligne s'évanouit en même temps que le mouvement et ce fantôme d'unité temporelle de l'espace se fond continûment dans l'espace intemporel ».

55.. On peut s'étonner que nous n'ayons pas évoqué ici le problème de l'animalité, comme étant une autre forme intermédiaire. Elle n'est pas inconcevable chez Sartre, même si elle est absente de L'Être et le Néant, selon l'aveu même de son auteur (voir l'entretien accordé en 1972 par Sartre, « Une vie pour la philosophie » repris dans Le Magazine Littéraire $\mathrm{n}^{\circ} 384$, février 2000 : «- Où sont les animaux dans l'Être et le néant ? - Ils n'y sont pas »). Cependant, le champ dans lequel se situerait cette problématique est bien plus celui de la conscience et des formes moins approfondies de néantisation que dans le champ de la nature pensée à partir de l'être. De fait, Sartre envisage les animaux, mais comme conscience. Pour lui, « je saisis que les animaux ont des consciences parce que je ne comprends leurs attitudes que si j'admets une conscience » (ibid.). La différence pourrait se situer dans le rapport à la contingence (voir les 
développements dans $C M$, p. 552-554). Il ajoute même que la conscience « c'est là où on la remarque, et il y a des animaux qui n'en ont pas, les protozoaires, par exemple. La conscience apparaît dans le règne animal à un certain moment, chez les hommes sûrement aussi chez les singes » (ibid.). Cette remarque ouvre le champ de l'analyse d'une relation à l'animal dont les Cahiers pour une morale donnent une esquisse en analysant le rapport à l'animal dans le cadre de la domestication : Voir CM, p. 331 : Mais cette liberté [de l'animal] n'est pas inquiétante car le chien n'est libre que pour m'adorer. Le reste est appétit, humeur, mécanisme physiologique ; en se détournant de moi, en grondant, il retombe dans le déterminisme ou dans l'obscure opacité de l'instinct ». En ce qui concerne l'animalité en nous, et, de manière plus générale, l'enracinement de l'homme au sein de la nature et dans l'être selon une structure de chiasme, la question ressortit à l'analyse de la facticité et de sa signification. La base de ce traitement se trouve cependant dans les options métaphysiques que nous définirons dans la partie suivante.

56.. EN, p. 237.

57.. Les différents aspects du rapport entre métaphysique et ontologie ont été étudiés par Jean-Marc MouilLIE dans le $\mathrm{n}^{\circ}$ 10/2002 de la revue Alter, "Sartre phénoménologue », p. 229-249, et synthétisés par Pierre VERSTRAETEN dans le Dictionnaire Sartre, François NOUDELMANN et Gilles PHILIPPE (dir.), Paris, Honoré Champion, 2004, article « Métaphysique ", p. 317-322.

58.. EN, p. 337.

59.. EN, p. 667. Il ne faut bien sûr pas entendre ce terme au sens trivial d'une genèse empirique. L'individualité dont il est question est celle des structures de révélation et de ce qui en elle, ne relève pas de l'analyse structurale : le surgissement du pour-soi n'est pas déductible de l'en-soi, le mouvement n'est pas déductible des catégories de la transcendance, et la structure " réflexive » du corps n'est pas déductible de la nécessité de l'inscription corporelle dans le monde.

60.. Notamment dans les $§ 1-2$ du chapitre consacré aux « Structures immédiates du pour-soi », EN, p. 112-120.

61.. Voir sur ce point, EN, p. 174-175.

62.. EN, p. 669.

63.. Voir $E N$, p. 669 : « C'est au métaphysicien que revient de décider si le « mouvement » est ou non une première tentative de l'en-soi pour se fonder ».

64.. EN, p. 671.

65.. EN, p. 673.

66.. EN, p. 477.

67.. EN, p. 673.

68.. EN, p. 673.

69.. La Gestaltpsychologie ou psychologie de la forme s'est développée au tournant du siècle à partir de certaines analyses de la perception et de la mise en évidence de phénomènes d'organisation structurant le champ perceptif. Cette mise en évidence a conduit à un transfert de ce modèle dans d'autres cadres. Sartre et surtout MerleauPonty connaissaient bien les travaux de cette école dont les principaux représentants furent Wolfgang Köhler et Kurt Koffka. Les travaux de la psychologie de la forme ont été présentés en France par Paul GuILlaume (La Psychologie de la forme, op. cit.).

70.. CM, p. 129. Il semble cependant n'user de cette notion de forme que pour l'action. Cela tient au fait que Sartre, dans la perspective morale qui est la sienne, a le souci de contrer le réductionnisme physicaliste dans son étude de l'action comme modification 
de l'en-soi. Ce faisant, tout en reconnaissant que la modification se fait selon le mode d'être de l'en-soi et est analysable, de ce point de vue, de manière analytique, il tient à marquer la dimension nouvelle de l'action humaine comme faisant intervenir quelque chose de neuf au sein de l'être dès lors qu'on se place au point de vue de la conscience, mais seulement si la conscience est active. Voir CM, p. 550 : «Quant aux modifications que le corps produit comme En-soi se produit dans l'être, elles sont toujours régies par la catégorie de l'extériorité d'indifférence : transférer, assembler, désassembler, mélanger, etc. Mais la conscience saisit ces modifications dans l'unité de formes ». Mais une chose est l'approche analytique, une autre est l'affirmation dogmatique que cette approche analytique résorbe totalement le sens de la Nature sur le plan ontologique de la relation originaire de l'Être au pour-soi. Si Sartre oscille par rapport à cette question, identifiant parfois la nature à la quantité et à l'extériorité (voir CRD, I, p. 186), dans une formulation hégélienne, il n'en reste pas moins que le contexte de ces formulations est celle d'un sens par rapport à la praxis dialectique, et que dans la même phrase, il pose également cette extériorité comme occasion de la totalisation.

71.. Voir M. Merleau-Ponty, Le Visible et l'Invisible, Paris, Gallimard, « Bibliothèque des idées », 1964, p. 312 : « Opposer à une philosophie de l'histoire comme celle de Sartre (qui est finalement une philosophie de la « praxis individuelle » - et dans laquelle l'histoire es la rencontre de cette praxis avec l'inertie de la « matière ouvrée », de la temporalité authentique avec ce qui la fige) [...] une philosophie de la structure[...]. Car l'histoire est trop immédiatement liée à la praxis individuelle, à l'intériorité, elle en cache trop son épaisseur et sa chair pour qu'il ne soit pas facile d'y réintroduire toute la philosophie de la personne ».

72.. Pour Merleau-Ponty, cette nature ne s'identifie pas à celle découverte par les sciences de la nature, mais renvoie, via une reprise du Lebenswelt husserlien, à un être originaire. C'est une problématique dont Sartre s'est toujours défié.

73.. [...] Le sensible, la nature, transcendent la distinction passé-présent, réalisent un passage par le dedans de l'un dans l'autre - Éternité existentielle. L'indestructible, le principe barbare/Faire une psychanalyse de la Nature : c'est la chair, la mère. (VI, p. 321) ; «Interprétation superficielle du Freudisme : il est sculpteur parce qu'il est anal, parce que les fécès sont déjà glaise, façonner, etc. Mais les fèces ne sont pas cause : si elles l'étaient, tout le monde serait sculpteur./ Les fèces ne suscitent un caractère (Abscheu) qui si le sujet les vit de manière à y trouver une dimension de l'être - / [Il] ne s'agit pas de renouveler l'empirisme (fèces imprimant un certain caractère à l'enfant). Il s'agit de comprendre que le rapport des fèces est chez l'enfant une ontologie concrète. Faire non une psychanalyse existentielle, mais une psychanalyse ontologique [...] (VI, p. 323-324). Cet aspect de la philosophie de Merleau-Ponty ne semble pas avoir été souvent étudié. Cependant, la consultation des inédits du philosophe a permis à Emmanuel de SAINTAUBERT dans Du lien des êtres aux éléments de l'être. Merleau-Ponty au tournant des années 1945-1951, Paris, Vrin, 2004, p. 225-320, de montrer le lien de Merleau-Ponty, non seulement avec cet aspect de la philosophie de Sartre, mais aussi avec ses sources d'inspirations, Bachelard et Ponge. E. de Saint-Aubert est critique par rapport au projet sartrien de psychanalyse existentielle.

74.. Voir « L'homme et les choses », dans Situations I, Paris, Gallimard, 1947.

75.. Francis PONGE, Le Parti pris des choses, Paris, Gallimard, 1943.

76.. Situations I, p. 270.

77.. Situations I, p. 266-267.

78.. Situations I, p. 267. Les exemples repris par Sartre de Ponge concernent l'eau (voir Situations I, p. 261-262).

79.. Situations I, p. 239. 
80.. Fût-ce en s'assimilant à lui, car la réduction de soi-même à l'extériorité est aussi une forme d'assimilation de l'en-soi au pour-soi, comme le masochisme est un essai de réaliser la totalité moi-autrui en me sacrifiant au regard d'autrui.

81.. Voir CM, p. 458.

82.. CRD, I, p. 196.

83.. EN, p. 113.

84.. Voir EN, p. 650 : « ... nous avons montré l'erreur qu'il y aurait [...] à croire que nous "projetons" nos dispositions affectives sur la chose, pour l'éclairer, la colorer ».

85.. Voir EN, p. 28, où Sartre refuse déjà l'assimilation de l'en-soi à la chose en soi kantienne et de sa thèse à l'idéalisme problématique de Kant.

86. Voir EN, p. 215, 217, 223, 254, 553-554.

87.. Voir EN, p. 272-273 : «C'est à la métaphysique de décider s'il sera plus profitable à la connaissance [...] de traiter d'un être que nous nommerons le phénomène, et qui serait pourvu de deux dimensions d'être, la dimension en-soi et la dimension pour-soi [...]». 88. Voir EN, p. 673 : « dans le cas où il paraîtrait utile d'employer la notion nouvelle de phénomène, comme totalité désintégrée, il faudrait en parler à la fois en termes d'immanence et de transcendance. [...] Mais l'immanence sera toujours limitée par la notion de transcendance et la transcendance par sa dimension de pour-soi ».

89.. EN, p. 669. On notera que le passage à la Critique n'abolit pas ce type de vocabulaire ni même ce type de spéculation, puisque Sartre y écrit que « l'histoire de l'Homme est une aventure de la Nature » (CRD, I, p. 186), formule indéniablement métaphysique, mais que la critique de la dialectique de la nature n'a pas empêchée, parce qu'elle surgit d'une structure de réciprocité (et non d'une causalité univoque) entre l'homme et la nature sous le signe d'une totalisation perpétuelle, l'histoire.

90.. EN, p. 348.

91.. EN, p. 399 : « Avoir des mains, avoir des mains qui peuvent se toucher l'une l'autre: voilà deux faits qui sont sur le même plan de contingence et qui, en tant que tels, relèvent de la pure description anatomique ou de la métaphysique ». Le fait d'être indiqué comme visible est plus complexe : tout dépend de la manière dont on comprend le terme visible. Deux significations sont possibles : soit celle d'être indiqué à vide par les choses dans les rapports d'ustensilité et dans la connaissance sensible, soit d'être vu et plongé parmi les choses, ce qui exige le surgissement du regard d'autrui et le développement de la relation à autrui.

92.. Voir EN, p. 222-225, p. 554, p. 646-662.

93.. Voir EN, p. 243-244, p. 552-553.

94.. EN, p. 669.

95.. Voir EN, p. 237 à propos des ustensiles.

96.. On pourrait exprimer autrement les choses en inversant le rapport de choix : le choix du monde peut se manifester comme choix de donner au mouvement le sens de montrer que tout ne se réduit pas au pour-soi, et qu'il y a une profondeur propre de l'être. La démarche de Merleau-Ponty nous semble procéder d'une telle logique.

97.. VI, p. 328.

98. Ibid.

99.. Voir Cahiers pour une morale, p. 57, à propos de la perception qui me révèle moimême comme chose et m'insère comme passif, en même temps que la passivité et l'activité, bannie au niveau des pures structures ontologiques de l'en-soi se trouvent introduites dans le monde : « La passivité est très exactement ma liaison avec l'En-soi ; ma liaison ontologique et pratique à la fois. Réciproquement l'En-soi devient actif 
puisqu'il menace. L'activité de l'En-soi est d'ailleurs uniquement destructive. [...] Le Pour-soi, en-soi néantisé, reste en-soi par rapport à l'En-soi. Il est la relation interne de l'En-soi avec lui-même (action) en même temps que l'existant par qui il se fait que l'Ensoi devienne un monde ".

100.. Cette formule se trouve dans les Cahiers pour une morale, tout en se situant à un niveau de radicalité analogue à celui de L'Être et le Néant, p. 501 : « dévoiler, c'est créer ce qui est ». On la retrouve dans le manuscrit publié sous le titre Vérité et existence, par A. Elkaïm-Sartre, Paris, Gallimard, « Essais », 1995, p. 20, p. 49.

101.. Les Cahiers pour une morale, qui rencontrent cette configuration, ne l'exploitent pas ainsi car le projet sartrien l'amène à faire ressortir ce qui est le plus propre à dévoiler la dimension créatrice de ce dernier, rejetant par contraste les structures naturelles dans l'interprétation déterministe.

102.. Quel est le rapport entre ce niveau et celui des analyses de la Critique ? S'il y a dans les deux cas l'introduction d'une dimension de passivité et notamment un retour des choses vers l'homme, la perspective de la Critique est différente, car elle s'inscrit dans une démarche critique et régressive. Deux aspects sont mis en évidence : le sens dialectique de la dimension de l'extériorité et de sa constitution dans le cadre de l'intelligibilité de la nature et la manière dont l'homme, comme organisme pratique se trouve pris, de par sa spécificité humaine, par les deux figures de la naturalité que sont l'immanence de la vie et les exigences du besoin. Dans ces deux figures se manifeste la spécificité de la praxis humaine et de la nature à l'intérieur et à l'extérieur de l'organisme pratique. Ce niveau s'avère important, voire essentiel car il montre la façon dont Sartre a traduit la limitation réciproque de l'immanence et de la transcendance. Cette traduction conduit à une reprise critique des perspectives sur la philosophie de la nature, critique qui doit être située dans le projet et les exigences qui sont les siennes. Le refus de l'affirmation dogmatique de la dialectique de la nature ne conduit ni à un pur subjectivisme, ni à une conception de la nature dans un matérialisme réductionniste. Au contraire, la prise en compte de la nature s'avère ici essentielle en ce qu'elle situe, et par le biais de la vie, et par celui de la matière, la praxis humaine dans sa spécificité et ses limites sans tomber dans le naturalisme (pour l'individu) ou dans l'organicisme (pour le groupe).

103.. Voir les notes de travail recueillies dans Le Visible et l'Invisible, p. 258-260.

104.. Merleau-Ponty, dès La Structure du comportement, Paris, PUF, 1941, s'oppose aussi bien à la réduction subjectiviste qu'à la transposition matérialiste qui pouvaient tenter les psychologues de la forme.

105.. On exclut ici l'animalité consciente, qui nous renverrait, comme nous l'avons indiqué plus haut, du côté du pour-autrui.

106.. Voir notamment $E N$, p. 622-626.

107.. EN, p. 646.

108.. Jean-Paul SARTRE, « Les mobiles de Calder », Situations III, p. 307-311, et plus précisément la fin de l'article : « En un mot, [...] [les mobiles de Calder] sont à la fois des inventions lyriques, des combinaisons techniques, presque mathématiques et le symbole sensible de la Nature, de cette grande Nature vague, qui gaspille le pollen et produit brusquement l'envol de mille papillons et dont on ne sait jamais si elle est l'enchaînement aveugle des causes et des effets ou le développement timide, sans cesse retardé, dérangé, traversé, d'une Idée » (p. 311).

109.. Voir MERLEAU-PONTY, VI, Notes de travail, p. 325-325. 
110.. Jean-Paul SARTRE, Carnets de la drôle de guerre, nouvelle édition augmentée du premier carnet par Arlette Elkaïm-Sartre, Paris, Gallimard, 1995, p. 362 (noté CDG dans la suite).

111.. CDG., p. 363.

112.. Sartre, dans les propos qu'il a pu tenir sur Merleau-Ponty, use de cette notion, comme par exemple dans Situations IX, Paris, Gallimard, 1972, p. 52 : « [L'appel de l'Être ] représente [...] pour moi une aliénation. Ça aussi on le sent chez Merleau-Ponty dans une certaine mesure : tout rapport rétrograde à l'Être, ou toute ouverture à l'Être qui suppose l'être à la fois derrière et devant l'ouverture comme conditionnant l'ouverture, ça me paraît comme une aliénation ».

\section{RÉSUMÉS}

L'auteur montre comment la question de la nature est abordable dans le cadre de la philosophie de Sartre. Il examine la pertinence et la portée de la réfutation de la dialectique de la nature présentée dans la Critique de la raison dialectique. Considérant l'analyse du mouvement dans L'Être et le Néant, il montre que le dualisme de Sartre ne doit pas être envisagé de manière simpliste : il peut laisser place à une approche philosophique de la nature.

The author will show how the question of nature can be dealt with the philosophy of Sartre. He questions the relevance of the refutation of the dialectic of nature which can be found at the begining of the Critique de la raison dialectique. By considering the analysis of movement in L'Être et le Néant he shows that Sartres ontological dualism should not be too strictly interpreted : it leaves room for a philosophical approach of nature.

\section{AUTEUR}

\section{LAURENT HUSSON}

Laurent Husson, né en 1962, ancien élève de l'ENS de Saint-Cloud, agrégé et Docteur en philosophie, est actuellement Maître de Conférences dans cette discipline à l'IUFM de Lorraine. À la suite de sa thèse, Dévoilement du monde et appréhension existentielle de l'être dans l'Être et le néant (Université de Paris XII, 2001), ses travaux portent sur l'ontologie sartrienne et ses possibilités, qu'elles aient ou non été développées par Sartre ainsi que sur la confrontation avec ses contemporains. Ils essaient ainsi d'en construire une interprétation philosophique structurelle. Il a participé à plusieurs ouvrages et numéros de revue (les Études sartriennes notamment) et à un ouvrage collectif sur Sartre, Jean-Marc MOUILLIE (éd.), Sartre et la phénoménologie, Fontenay-aux-Roses, ENS Éditions, 2000. 